\title{
Research on Mechanism of Stock Bubbles Based on Bubble Dynamics
}

\author{
LI Feng \\ College of International Business, Shenyang Normal University \\ Shenyang, China \\ email:lifeng670312@sina.com
}

\begin{abstract}
Definition of Bubbles in economy originated from physics due to their similarities in features and evolution mechanism, which therefore makes it necessary to imitate and bring back law of stock trading through the origination of bubbles based on related research results on bubble dynamics. A great deal of relevant studies makes it clear that financial capital naturally presents some bubble features. Financial capital resembles bubbles of thermology in formation mechanism and character. Bubbles and bubble mechanism have been studied profoundly in physical thermology. Study of bubble in physical thermology has achieved maturity. Hence, with the aid of some research methods and results on bubble dynamic of physical thermology, this paper intends to study formation law, expansion, atrophy, deformation and collapse of stock bubble, hopefully to achieve certain satisfactory results.
\end{abstract}

\section{Keywords- bubble;dynamic; stock;formation law;mechanism}

\section{ANALYSIS OF FORMATION PROCESS OF BUBBLE AND FOAM BASED ON BUBBLE DYNAMICS}

Air or other substance of gas is pressed under the surface of liquid which starts to expand. Liquid membrane then seals up air to form bubbles. Owing to the density difference between air and liquid, bubble is easily to float up to the surface of liquid. So these bubbles gather together and develop constitution of bubbles which is split up by liquid membrane. Constitution of bubbles meets the definition of foam. Foam is a certain kind of dynamic system in thermodynamics. As foam burst, surface area and energy goes down as well. Exploding of foam can be interpreted like this: the liquid membrane which separates liquid and air first blows much thinner and finally burst. One of the major factors of foam blow can be attributed to pressure difference between inside and outside of bubble. No matter whatever factors, pressure difference plays a key role to make it possible.

\section{A. Mechanisms of formation, expansion and collapse of individual bubble}

Formation of bubble is owing to exterior work. When exterior work reaches certain amount, bubble comes into birth and then energy of the whole system goes up. As to the formation of bubble, surface between air and liquid increase dramatically. Bubbles composition can be defined as dynamic bubbles composition in thermology for its increase in energy. Surface extension of bubble surface is interior contractile force which can cause the decrease of energy so as to reach the stability of bubbles composition.
Bubble membrane is under force from three parts. The first one is surface expansion. The second one comes from the pressure difference between outside medium such as air and force $\mathrm{p} \pi \mathrm{r} 2 . \mathrm{P}$ in $\mathrm{p} \pi \mathrm{r} 2$ is added pressure which is pressed on liquid membrane through its bottom. Area of bottom is $\pi \mathrm{r} 2$. The last one is gravity on liquid membrane which is minor force compared the first two force and then it can be ignored. The first and second force can be hypothesized as $f_{1}$ and $f_{2} . f_{1}$ is interior contractile surface tension force to make the system remain stable, $f_{1}=\alpha L \quad f_{2}$ is exterior expansion force, which is the result difference in intensity of pressure, $f_{2}=p \pi r^{2}$. Interior intensity of pressure is bigger than the exterior. $f_{2}$ is developed due to the work pressed on formation of bubble. When $f_{2}$ is stronger than $f_{1}$, bubble expands and liquid membrane becomes thinner. In the expanding process of bubble, difference of intensity of pressure is narrowing down and attenuation, namely reduction of $\Delta P$.Expansion force towards outside gradually decreases.

While, at the same time, surface tension $f_{1}$ magnifies following the growing of bubble. The reason is that $L f_{1}=\alpha L$. Expanding of bubble causes $L$ increases and then finally $f_{1}$ rises. Speed of bubble expansion reduces step by step due to the increase of $f_{1}$ and decrease of $f_{2}$. When the two forces reach the same amount, bubble stops its expansion. At this moment, area of bubble reaches its maxim, as well as the surface area. Bubble is the most venerable due to its much thinnest liquid membrane. It is sure that the least interference could even cause major destroy.

\section{B. Mechanism of formation, expansion and collapse of multi-bubbles}

Foam is bubble composition. When certain amount of bubbles develops, its speed and size can not be same because of the difference on time and work when they formed. Intensity of presser in small bubble is bigger than in big bubble. Therefore, the small bubble discharges air to big bubble through liquid membrane for difference of intensity of pressure. At the same time, energy is transferred into big bubble. This process can be termed as work on big bubble. Small bubble shrinks to disappear during the discharge processes. On the contrary, big bubble expands so much that liquid membrane collapse. 
Reexamining the bubble composition, we may discover that single bubble in bubble composition do follow the same routine as the individual bubble. Discharge of air can be applied both to the big and small bubble. At last, process of discharging air of both big and small give birth to the big bubble which shares the same routine of formation, expansion and collapse with individual bubble. Certain amount of individual bubbles composes foam composition. Formation, expansion and collapse of multi bubbles make them possible to develop to be a big bubble which expands to certain degree and then collapse. This also can be defined as the collapse of foam. It can not be denied that collapse of individual bubble of does exist. But the truth is that collapse of individual bubble can not be influential enough to lead to the chain reaction of collapse of other bubbles.

\section{STUDY OF STOCK BUBBLES TRAINNG MECHANISAM BASED ON BUBBLE DYNAMICS}

\section{A. Analysis of formation of stock bubble}

Stock investment can be fulfilled by interest and profit of stock transference. The major one comes from added value through stock transference. The added value indicates the starting point of bubble in stock market. Energy of stock expansion is an influence on stock bubble.

Energy of stock can be impacted by multi factors which include the financial situation, trading activity and expectation of trading market. The most influential factor is stock price and trading volume. Here we can name stock energy $E$, volume of stock transference as $V$, stock price as $P$, to imitate energy formula in thermal science, we can define stock formula as the following:

$$
E=P V
$$

We can interpret stock energy as the amount of transaction of individual stock.

With the expansion of stock bubble, stock price, volume of transaction increase from the $P_{1}, V_{1}$ at the moment of $t_{1}$ to $P_{2} 、 V_{2}$ at the moment of $t_{2}$,

Total amount of energy of stock equals to the following:

$$
E=\sum_{t=t_{1}}^{t_{2}} P V
$$

In certain amount of time, grow of stock price transaction, frequency of transaction and trading could all contribute to the accumulation of interior energy of stock and enhance the expansion of stock bubble. We may have the hypothesis like this: at the moment of ${ }^{t_{3}}$, stock attains added capital $M$, total energy of stock is

$$
E_{3}=E+\alpha M
$$

In this formula, $\alpha$ is coefficient as capital changed to added energy. This coefficient grows bigger with the increase of trading activity. Under the push of added energy, price of stock and volume of trading would $\operatorname{reach}_{P_{3}, V_{3}}$,

With the support of energy, stock bubble would present the following trend: a. Initial period: the energy remains at the relatively bottom level because stock price, volume of transaction and trading activity are all being low. Energy of stock bubble is also limited. If stock keeps going for certain period of time, trading of stock would remain inactive in the long run.

b. Mild expansion of stock bubble: bubble expansion is also the consumption of energy. When the total amount of capital of stock is fixed, expanding of stock could only rely on the increase of stock transaction. Increase of stock transaction would promote the energy of stock to increase gently. But we have to admit that this gentle increase could only provide limited energy. When bubbles expand to certain degree, expansion will suspend without involvement of new energy as bubbles reach balance. We should notice that this balance is constantly instable, even the minor disturbance would break the balance.

c. Booming of stock bubble: formation of bubble is caused by the exterior force at one-off. In contrast, stock bubble could only develop when new investors of stock, under the influence of the successful profit winner, start to get involved in stock transaction.

Promoted by added capital and active stock transaction, stock bubble booms dramatically. As stock energy accumulates sharply, stock would expand wildly and lose stability without appropriate way to leak out. Finally the unlimited expansion will lead to the collapse of stock bubble.

\section{B. Analysis of stock stability}

As an instant thermal dynamics, stability of bubble lies on the change of intensity of pressure both inside and outside. Similarly, stock bubble also shares the same routine to expand, with the liquid membrane becoming thinner and thinner and then bubble burst. Its stability also rests on difference of intensity of pressure.

Interior tension is a kind of force towards outside. It has positive correlation with profit expectation and instant capital investment. It is therefore has negative correlation with range of expansion, namely price of stock. We could easily conclude that the higher the stock price is, the lower the interior tension would be.

Here we could regard interior tension as $f_{1}$, profit

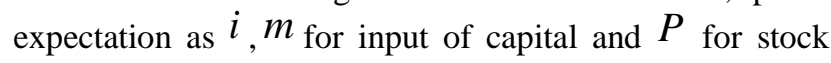
price

We may have the formula of interior tension:

$$
f_{1}=F(i, m) / P
$$

Exterior force is a contractile force which has positive correlation with macro economy and self value of stock.

Exterior force is $f_{2}, v$ for the own value of stock, $\alpha$ as macro economy

Formula of exterior force:

$$
f_{2}=F(v, \alpha)
$$

$F$ is difference of intensity of pressure between interior and exterior force

$$
F=f_{1}-f_{2}
$$

Under the pressure coming both from outside and inside, stock bubble would present the following states: 
a. $f_{1}=f_{2}$, when they remain the same, stock bubble keep the instant stability. At the critical edge, stock bubble stays extremely dynamical state. Surprisingly, even the minor disturbance could break balance leading to the trend to opposite direction. As for the stock exchange, it is a relatively stable period, with possibility both up and down.

b. $f_{2}>f_{1}$, when exterior goes strong then interior force, stock bubble presents negative bubble. Under the exterior force, stock bubble totally would shrink and then collapse. Stock transaction goes down and then might suspend facing the challenge of delisting. Government would take severe deflation policy. It is also possible that the stock loses its brand value.

c. $f_{1}>f_{2}$, when interior force goes stronger, bubble would expand greatly as it surpasses exterior force, bubble presents positive state to expand constantly. Difference of intensity of pressure between interior and exterior is decreasing and in the state of attenuation with the expansion of bubble, namely, the reduction of $\Delta F$. Tension towards outside is also becoming smaller. In other words, $f_{1}$ is reducing. While, exterior force $f_{2}$ enlarges with the expansion of bubble. Speed of expansion would slow down gradually. When the interior and exterior forces reach the same level, bubble stops its expansion. Bubble reaches its maxim in size at the moment as well as the surface area. Stock bubble reaches its summit. But the liquid membrane is the least thin. That is to say it is mostly like to explode. Even the minor disturbance would make it happen. It is avoidably to explode sooner or later.

\section{Analysis of mechanism of collapses of stock bubble}

As an instable composition, stock bubble will doom to collapse under the following situations

a. appropriate collapse

Stock bubble is doomed to collapse as an instable composition. The big difference lies in the period for individual stock. Each individual stock would go through the period like blossom, summit and collapse. Remaining long stability is rarely to be seen in stock market. Commonly, the longer the stock bubble remains, the ratio of expansion would be bigger. In such circumstance, liquid membrane is becoming thinner as well as the decrease of its intensity. Generally, this stock bubble is under extremely instable situation. Any minor disturbance such as rumor or changing of policy trend would be disastrous for it. The result would be even worse. The return period will go relative longer. So, it is wisely to restrain inflation of stock, which can be a reasonable step to hinder the collapse of stock market.

b. puncture of stock bubble

Puncture of stock bubble is to adapt manual measure to regulate balance to force it collapse in advance. In critical moment, state government would take firm policy to restrain over inflation prevent economic bubble. Stock with lower self-value and flexibility to resist exterior presser would collapse first. This kind of stock is often the junk stock. We have to admit that compelling to disturb the period of stock would inevitably cause side-effect. While, in more general sense, it is worthwhile to forcefully make certain stock collapse as to pay the price for restrain the overheating of state economy.

c. collapse of reconciled stock

When individual stock bubble remain stable, reconcile of stock bubble could also lead to the collapse. Stock merging could result in reconciles of stock bubbles. Reconcile, known as the positive stimulation, could speed up the expansion of bubble. It is therefore possible to collapse.

\section{ANALYSIS OF MECHANISM OF STOCK BUBLE BASED ON BUBBLE DYNAMICS}

In stock market, individual stock bubble would not be able to trigger stock bubble entirely. But, we have to be aware of the irrational factors. For example, herd effect and herd mentality drive investors to behave blindly and wildly. Financial income is a great influence currently. Individual stock bubble would be quite easily to initiate multi stock bubbles and finally spread to the whole stock market.

Stock bubble could be simply regarded as the composition of individual stock bubble. Integration of several individual stock bubbles may trigger the following situations:

First of all, several stock bubbles integrate to form big individual stock bubble. Each stock bubble stimulates and drags which can help to enhance speed of stock expansion for financial effect. Gradually, these stock bubbles form a big individual stock bubble which contains the original energy and presents trend of high speed expansion. Finally, the bubble of stock market presents. Under the normal situation, that is to say without outside manual interference, it is naturally to see the collapse of bubble of stock market.

The next worth mentioning is symbiosis of several stock bubbles. When several medium level stock bubbles integrate, they will grow harmoniously on their own but not depend on each other. As each one of them is relatively small without severe difference on size and thick liquid is rather thick, when they gather together they could be isolated among each other. For the stock market, this symbiosis can be simply regarded as the composition of several small stock bubbles. Even the collapse of individual bubble would not cause the chain reaction to other bubbles. The whole remains at a gently expanding state, which can be termed as sub-stock bubble period More attention is needed without concern or even worrying it.

\section{CONCLUSIONS}

This paper applies bubble dynamics to analyze bubble in stock market. Certain conclusions have been reached.

A. Stock bubble is assumed as energy composition. Stock energy could promote expansion of stock constantly till its collapse.

B. Stock bubble can be seen as an unstable composition. Difference of intensity of pressure is an influence on its stability.

C. Increase of $P_{2}$ is the influential factor of stock expansion even collapse. It is necessary to restrain the increasing speed of stock price.

D. As for the stock bubble, to extend the width of liquid membrane, namely the sustainability of stock and its 
profit could control speed of expansion and delay the time of collapse.

E. Collapse of stock can be divided into three ways: self collapse, puncture and reconcile.

F. Bubble in stock market can be regarded as the composition of several stock bubbles. When several individual stock bubbles accumulate, they can form the individual big stock bubble or symbiosis of several stock bubbles.

\section{REFERENCES}

[1] J. Duan, Study of Chinese Stock Bubble, Changsha: Hunan University, 2002.

[2] X. Q. Ge, Theory of Stock Bubble and Model, Beijing: Economy \& Science Press, 2005.

[3] K. Zhang and D.W. Su, "A Study on Bubbles Measurement and Bubbles Rationality in China's Stock Market," The Theory and Practice of Finance and Economics, vol. 28, Jan. 2007, pp.34-39.
[4] S. Shuo, Japanese Financial Policy, Tokyo: Iwanami Shoten, 1993.

[5] R. S. Gurkaynak, "Econometric tests of asset price bubbles. Taking stock, "Journal of Economic Surveys,:Vol. 22, 2008, pp. 166-186.

[6] M. Yoshihito, Coping Strategies of Stock Economy - Theory of Economic Recession, Beijing: Press of People's University of China, 2000.

[7] H.Ulrich and B. Jörg, "Testing for Speculative Bubbles in Stock Markets: A Comparison of Alternative Methods," Journal of Financial Econometrics,vol.10, Issue 1, Winter 2012, pp. 198-231. doi: 10.1093/jjfinec/nbr009.

[8] H. Helmut and K. A. Kholodilin, "In-Sample and Out-of-Sample Prediction of stock Market Bubbles: Cross-Sectional Evidence", Journal of Forecasting, vol. 33, Issue 1, Jan. 2014, pp. 15-31, doi: 10.1002/for.2269.

[9] C. P. Kindleberger, Manias, Panic and Crashes-History of Financial Crisis, Beijing: China Financial Press, 2007.

[10] J.Cuñado,L. A.Gil-Alana, and F. Perez de Gracia, "Testing for stock market bubbles using nonlinear models and fractional integration," Applied Financial Economics, vol.17, Issue 16, Nov. 2007, pp.1313-1321. 